\title{
Fabrication of on-chip probes for double-tip scanning tunneling microscopy
}

\author{
Maarten Leeuwenhoek ${ }^{1,2}$, Freek Groenewoud ${ }^{2}$, Kees van Oosten ${ }^{2}$, Tjerk Benschop $\mathbb{D}^{2}$, Milan P. Allan $\mathbb{D}^{2}$ and \\ Simon Gröblacher (iD)
}

\begin{abstract}
A reduction of the interprobe distance in multiprobe and double-tip scanning tunneling microscopy to the nanometer scale has been a longstanding and technically difficult challenge. Recent multiprobe systems have allowed for significant progress by achieving distances of $\sim 30 \mathrm{~nm}$ using two individually driven, traditional metal wire tips. For situations where simple alignment and fixed separation can be advantageous, we present the fabrication of on-chip double-tip devices that incorporate two mechanically fixed gold tips with a tip separation of only $35 \mathrm{~nm}$. We utilize the excellent mechanical, insulating and dielectric properties of high-quality SiN as a base material to realize easy-toimplement, lithographically defined and mechanically stable tips. With their large contact pads and adjustable footprint, these novel tips can be easily integrated with most existing commercial combined STM/AFM systems.
\end{abstract}

\section{Introduction}

Scanning tunneling microscopy (STM) that uses two tips simultaneously, also called double-tip STM, relies on two individually driven metal wire probes brought into close proximity to locally probe the resistivity ${ }^{1-3}$ or access proposed electron correlations at the nanoscale ${ }^{4-11}$. Achieving tip separation down to the nanometer scale, a long-standing goal in multiprobe STM, has proven challenging and is limited by the radius of curvature of the two tips $^{12}$ and requires sophisticated navigation routines ${ }^{13,14}$. Recently, multiprobe systems able to achieve tip separation down to $30 \mathrm{~nm}$ have emerged ${ }^{1,13}$ and have resulted in the first double-tip correlation measurements to date ${ }^{15}$.

These experiments, however, must undergo complicated alignment procedures and are limited to specialized STM setups. Here, we continue to build on earlier work ${ }^{16}$ to create a robust and easy-to-implement on-chip solution where both tips are integrated on a silicon chip. With

\footnotetext{
Correspondence: Milan P. Allan (allan@physics.leidenuniv.nl) or Simon Gröblacher (s.groeblacher@tudelft.nl)

${ }^{1}$ Kavli Institute of Nanoscience, Department of Quantum Nanoscience, Delft University of Technology, Lorentzweg 1, 2628CJ Delft, The Netherlands ${ }^{2}$ Leiden Institute of Physics, Leiden University, Niels Bohrweg 2, 2333CA Leiden, The Netherlands
}

our approach, given the joined nature of the tips, we can eliminate the need for an additional scanning electron microscope column ${ }^{13,17-21}$ for navigation and make this approach compatible with ultrastable compact Pan-type STM heads widely used for single-tip experiments ${ }^{22}$. Moreover, the millimeter-scale contact pads and adjustable footprint allow for easy integration in existing and commercially available STM systems. Existing on-chip scanning probes have already contributed to several techniques, such as parallel $\mathrm{AFM}^{23}$, scanning near field microscopy ${ }^{24}$, scanning Hall probes ${ }^{25,26}$, and scanning SQUID probes ${ }^{27}$. However, the development and use of integrated STM tips has been limited ${ }^{28-31}$. Recent proofof-principle experiments have demonstrated that such single-tip probes can meet the stringent criteria for $\mathrm{STM}^{29}$ even under ultrahigh vacuum and low-temperature conditions ${ }^{16}$.

The main challenge in realizing multitip STMs is to minimize the tip-to-tip distance while maintaining the excellent stability required for prolonged in- and out-offeedback measurements to obtain high-quality topographic and spectroscopic data. The double-tip devices presented in this letter build on the recently introduced SiN-based smart tip platform ${ }^{16}$ and now incorporate

\section{(c) The Author(s) 2020}

(c) (i) Open Access This article is licensed under a Creative Commons Attribution 4.0 International License, which permits use, sharing, adaptation, distribution and reproduction cc) in any medium or format, as long as you give appropriate credit to the original author(s) and the source, provide a link to the Creative Commons license, and indicate if changes were made. The images or other third party material in this article are included in the article's Creative Commons license, unless indicated otherwise in a credit line to the material. If material is not included in the article's Creative Commons license and your intended use is not permitted by statutory regulation or exceeds the permitted use, you will need to obtain permission directly from the copyright holder. To view a copy of this license, visit http://creativecommons.org/licenses/by/4.0/. 
high-resolution focused ion beam (FIB) milling to achieve nanometer tip separation while maintaining a rigid connection through a thin silicon nitride ( $\mathrm{SiN}$ ) support. The combination of the mechanical stability provided by the SiN platform with high-resolution milling yields a unique and straightforward approach to the fabrication of scanning probes and their future use in double-tip STM.

\section{Results}

Figure 1 gives an overview of several devices we fabricated using the methods described above. Figure 1a shows an overview of the chip, where the three contact pads separated by trenches can clearly be identified. The narrow bright lines along the pads are the regions consisting of undercut SiN covered in gold, like the rest of the chip. The angled view clearly shows the sidewalls of the chip and the overhanging tips where the contact pads meet.
High-magnification images provide a detailed view of the apices of the tips, where Fig. $1 \mathrm{~b}$ was taken before the FIB step. Such a device can act as a single-tip device or potentially be used to perform tip preparation through heating by running a current between the contacts, as suggested by Ciftci et al. ${ }^{32}$.

Figure 1c, d shows the apices of two tips from the front under a $52^{\circ}$ angle and from the top, respectively. They allow us to clearly see the metal film coverage on the sides of the SiN layer as well as the separation between the tips, which was $\sim 35 \mathrm{~nm}$ edge-to-edge. The relatively thick $\mathrm{Au}$ film became grainy, especially near the apex of the $\mathrm{SiN}$, while it was smoother on the pads. Even though the coarseness of the metal made the exact location of the tip uncertain, the metal consistently covered the full apices (Fig. 1d). For this particular device, the FIB milling depth was set to $300 \mathrm{~nm}$ to ensure a cut through all the metal on the side of the SiN.
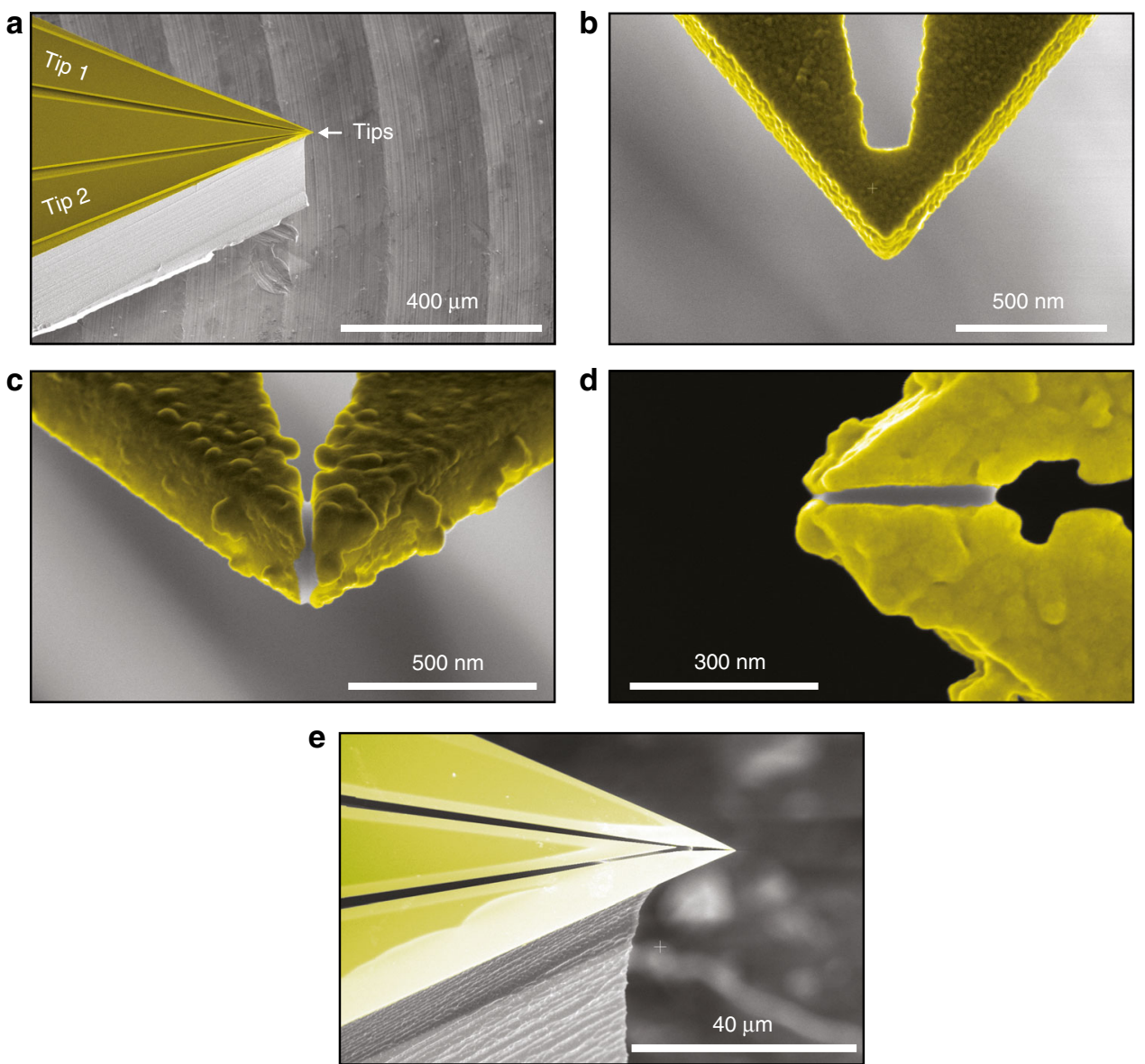

Fig. 1 Colored SEM images of several double-tip devices after deposition of Au (yellow). a Tilted profile of the device showing the profile of the chip and the protruding tips. $\mathbf{b}$ Tip apex before FIB milling. c Tilted front view of the two tips after FIB milling. The two tips originated from a $55 \mathrm{~nm}$ gold layer, and the gap between the tips was $\sim 35 \mathrm{~nm}$; hence, the total distance between the points that were most likely to tunnel was $\sim 50 \mathrm{~nm}$. The required 3D alignment can be directly estimated from these tilted images. $\mathbf{d}$ Top view of the same device as (c) shows clear protrusion of the metal film beyond the SiN. e Side view of the device shown in (c) and (d). 


\section{Discussion}

The general fabrication process herein follows the process for SiN-based smart tips introduced earlier for single-tip devices ${ }^{16}$. Here, we continue to build on this platform as we extend the fabrication procedure to create double-tip devices by incorporating several new techniques. The high-quality $\mathrm{SiN}$ that provides the base for the tips plays a central role in the design and fabrication of the devices. First, their excellent insulating, dielectric and mechanical properties provide the opportunity to keep the two tips mechanically attached while electrically separated. Second, the $\mathrm{SiN}$ also enables the use of a single lithography step that incorporates the additional complexity that the multiple tips and contact pads bring. By transferring the shape of both tips and their contact pads into the $\mathrm{SiN}$, we can later use an isotropic silicon etch to create trenches that electrically separate the tips even after deposition of the metal, as we will show below.

\section{Pattern design}

A schematic overview of the full fabrication procedure is shown in Fig. 2. We start with a $200 \mathrm{~nm}$ thick layer of high stress silicon nitride deposited with low-pressure chemical vapor deposition (LPCVD) on both sides of a $200 \mu \mathrm{m}$ thick Si (100) chip, and we deposit a $550 \mathrm{~nm}$ thick layer of ARP-6200.13 resist by spin coating on top for electron beam lithography. The pattern is created by a single exposure but consists of two parts. The first is the tip shape itself with the surrounding shields, which we include to minimize the overhang of the SiN surrounding the tip after the Si etching process, as described below. By covering these areas with the shields, the isotropic etch only affects the sidewalls and does not create a significant undercut from the top. The second part consists of the two contact pads and a center pad that is used to reduce the exposed area to shorten the exposure time and minimize the proximity effects of the exposure near the two tips. For future applications, this part can also act as a third contact pad (combined AFM/STM systems often include three electrical contacts). The large areas are exposed by a $40 \mathrm{~nm}$ electron beam with a beam step size of $20 \mathrm{~nm}$ and a dose of $400 \mu \mathrm{C} / \mathrm{cm}^{2}$, and the small structures are exposed by a smaller beam with a size of $18 \mathrm{~nm}$, a step size of $2.5 \mathrm{~nm}$ and a dose of $320 \mu \mathrm{C} / \mathrm{cm}^{2}$, both with a $100 \mathrm{kV}$ beam. Note that the narrow trenches between the tips/contacts could lead to electrical shorts formed by accidental left-over traces of resist inside the trenches or small pieces of dirt that connect the pads after deposition of the metal. Therefore, we enlarge the spacing between the tips and pads as we move away from the apices of the two tips that are still connected at this stage.

We then developed the chip in pentyl acetate $(1 \mathrm{~min})$ and MIBK:IPA 1:1 (1 min) followed by an IPA rinse (1 min), resulting in Fig. 2a. The pattern, consisting of the tip shape and six shields, is transferred into the SiN layer using a $\mathrm{CHF}_{3}$ etch for $5 \mathrm{~min}$ (Fig. 2b). After we start the removal of the resist by exposing the chip to an $\mathrm{O}_{2}$ plasma for $10 \mathrm{~min}$, we then continue cleaning the chip by successive immersions into N-N-dimethylformamide (DMF) for $10 \mathrm{~min}$, a positive resist stripper (PRS) for $10 \mathrm{~min}$, followed by a boiling piranha solution at $135^{\circ} \mathrm{C}$ for $8 \mathrm{~min}$ to remove all traces of the resist and other organic contaminants (Fig. 2c).

\section{Dicing}

To bring the tip to the edge of the chip, we proceeded with dicing (Fig. 2d). Here, we prefer dicing over a through-wafer deep reactive ion etch (DRIE) since it allows us to easily explore different designs with each new iteration and reduces the number of fabrication steps. We would, however, like to stress that our method is fully compatible with full wafer processing, as we discuss later.

Before the dicing step, we first protect the chip surface against any residual debris by applying a new layer of photoresist. From a typical $10 \times 10 \mathrm{~mm}^{2}$ chip, we cut two smart tips to ensure that the small features of the tip are in the center of the chip for optimal resist conditions for EBL (Fig. 3c). Successful dicing results in (i) smooth sidewalls, such that the overhanging tip is the most protruded feature, (ii) there is minimal chipping of the Si and, (iii) there is good alignment accuracy.

The first requirement is obtained by choosing the optimal blade and settings for the dicer (Disco dicer DAD 3220). The Disco ZH05-SD2000-N1-90 blade at a forward speed of $3 \mathrm{~mm} / \mathrm{s}$ yields optimal results in terms of chipping and sidewall smoothness. The limited residual roughness on the sidewall is further smoothed out by the isotropic Si etch described in the next section.

To align the dice consistently down to a few microns from the patterned tip, we minimize the amount of chipping of the Si along the dice line (Fig. 3b). The blade is therefore typically dressed - resharpened by cutting into a special substrate and increasing the exposure of the diamonds in the blade for each new chip (two tips), and we perform the most critical dice (number 1 in Fig. 3a) first, as the chipping increases with each dice.

Last, to align accurately to the patterned tips, we calibrate the width of the blade with a so-called hairline adjustment.

\section{Suspending SiN tip}

First, we wash away the residue created by dicing with the removal of the protective photoresist layer, and then the chip is cleaned again with a 10 min $\mathrm{O}_{2}$ plasma. After these cleaning steps, we isotropically remove part of the $\mathrm{Si}$ substrate by exposure to an $\mathrm{SF}_{6}$ plasma for 3-4 min inside an inductively coupled plasma (ICP) etcher. Reactive ion etching is often a combination of chemical reactions, 

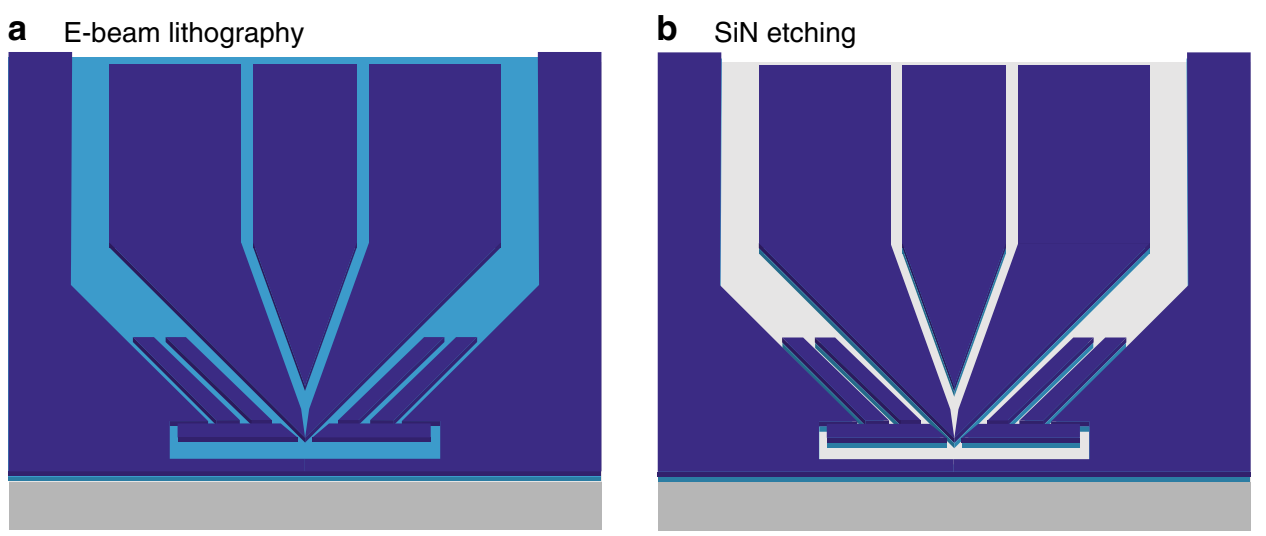

C Resist stripping

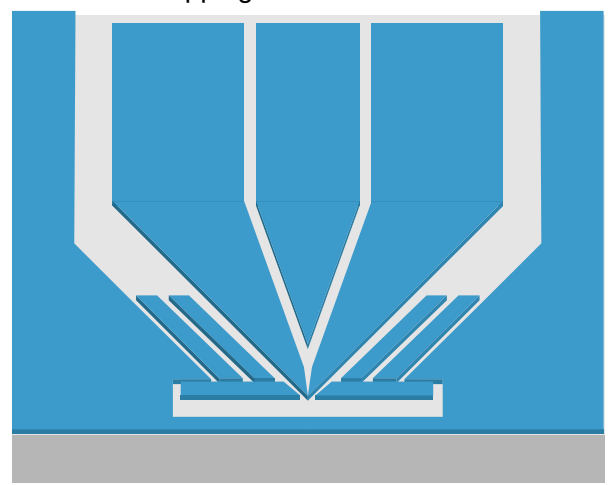

e Si etch

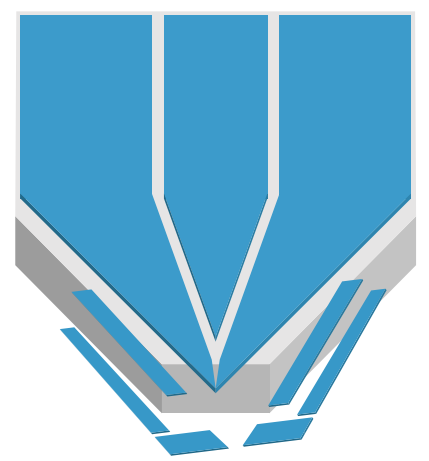

d Dicing

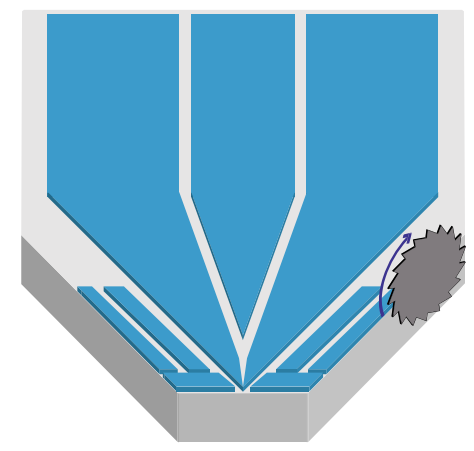

f Metallization + FIB

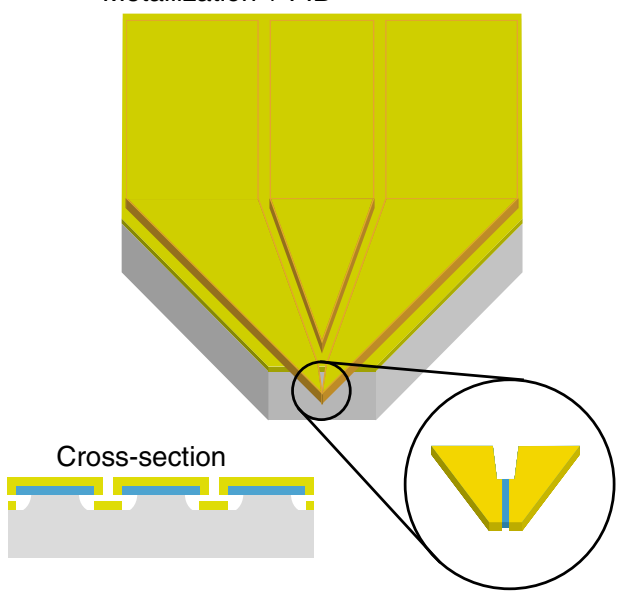

Fig. 2 Fabrication procedure for double-tip devices. a The resist layer (purple) on the SiN (blue)-covered Si (100) chip (gray) is patterned by e-beam lithography, creating a large opening in front of the tips, around the contact pads, and along the lines that encircle the shields and the tips. b The pattern is then transferred into the SiN. c The resist is cleaned thoroughly, and a fresh layer of photoresist is applied to protect against debris from dicing (not displayed for clarity). $\mathbf{d}$ The chip is then diced (details can be found in Fig. 3). e After removal of the protective resist, the chip is undercut using an isotropic Si dry etch that removes the Si substrate primarily from the sidewalls causing the shields to drop off, leaving the tips freestanding. $\mathbf{f}$ We evaporate a Au layer on top of a Cr adhesion layer followed by focused ion beam milling to separate the two tips (inset right). The electrical isolation of the contact pad is illustrated in the cross-section inset.

where $\mathrm{F}^{-}$ions react with $\mathrm{Si}$, and ion bombardment, where the ions are accelerated by a bias into the sample, which removes material. Here, however, to prevent any anisotropy in the etch, we apply a bias except for during a short ignition pulse $<1 \mathrm{~s}$ to ignite the plasma, resulting in a predominantly isotropic $\mathrm{Si}$ etch $^{33}$. To increase the selectivity of the $\mathrm{SiN}$ over the $\mathrm{Si}$, the chip is cooled to $-50^{\circ} \mathrm{C}$. 


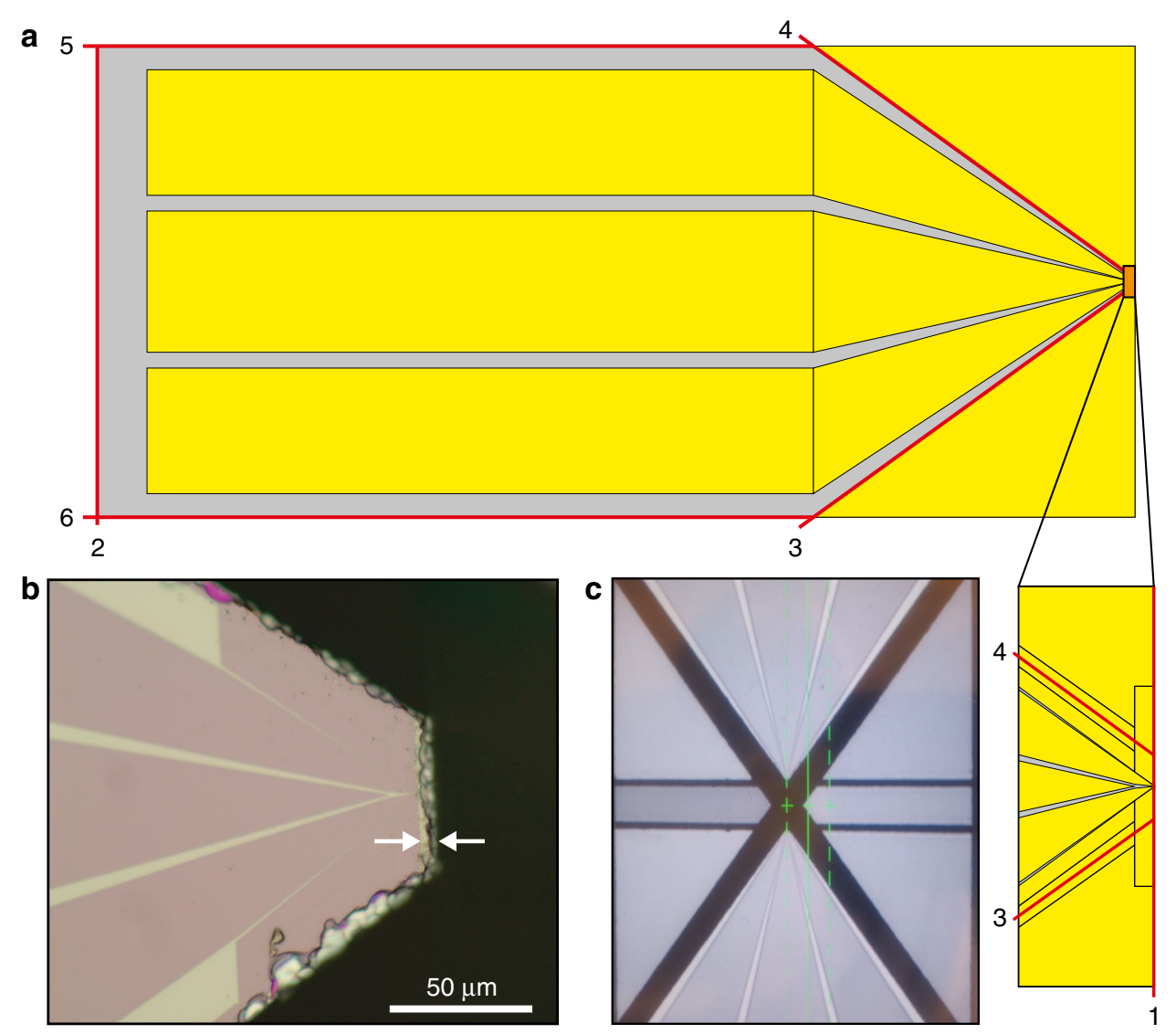

Fig. 3 Double-tip device design. a Double-tip pattern design with dice lines shown in red. The gray areas indicate Si, while the yellow parts are covered with SiN. The small features, such as the tips and the surrounding shields, are highlighted in the inset on the bottom right. b Optical microscopy image of a chip after dicing. The silicon (gray) left after the dicing extends up to $6 \mu$ m beyond the SiN (pink). c Screen capture of a chip containing two opposing smart tips after dicing as seen through the microscope on the dicer. For illustrative purposes, the image is taken at low magnification, and the alignment of the blade is performed at high magnification. The black areas are diced.

\section{Metallization}

After the overhang is created, we proceed to deposit the metal film. While our previous work ${ }^{16}$ relied on sputtered $\mathrm{Au}$ films to ensure maximum coverage of the SiN sidewalls, here we use an electron beam evaporator to deposit a 3-5 nm $\mathrm{Cr}$ adhesion layer and a $45-60 \mathrm{~nm}$ thick $\mathrm{Au}$ film. The evaporation of Au films is more directional but should in combination with a $\mathrm{Cr}$ layer underneath show improved adhesion to the SiN. To counter the effects of the directionality of the evaporation process compared to that of sputter deposition, we place the chip at an angle of $\sim 40^{\circ}$ and rotate it at $10 \mathrm{rpm}$ to obtain an appropriate sidewall coverage.

\section{Optimizations and challenges}

Before demonstrating the final devices, we employ the optional optimization. If the overhangs created on all edges break, shorts to the lower lying metal on the Si can occur, especially during mounting in the STM tip holder.
To date, both the protrusion of the tips and the overhanging sides of the contact pads have been created with the same isotropic Si etch. We do this after dicing and subsequent removal of the protective photoresist with acetone and IPA. However, we can also choose to leave the resist used during dicing on and perform the etch, as illustrated in Fig. 4. Now only the sidewalls of the chip retract while the top layer is protected, therefore we create a protrusion of the tip, but no trenches between the contact pads. The latter, however, is needed to create electrical separation of the pads. Once the tip overhangs and (almost) all shields are held by the resist, we spray acetone and IPA to clean off the resist in the direction away from the tip to prevent the shields from landing on top. Finally, we can perform a very short additional isotropic etch to form the contact pads. The result is smaller overhanging regions around the sides of the contact pads. The decrease is determined by the length of the last etch. 
a

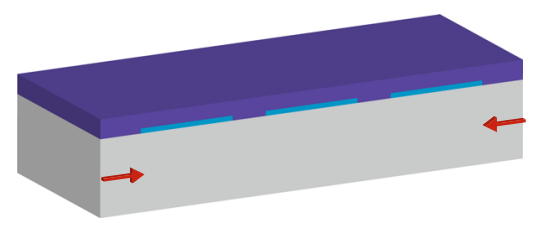

b
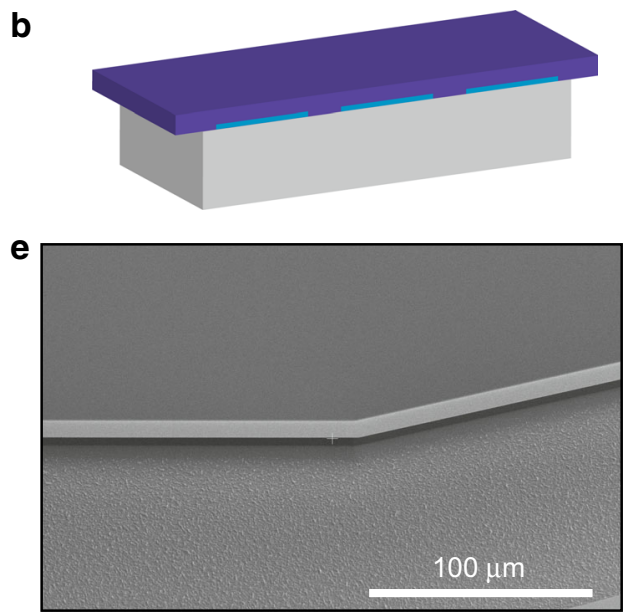

C

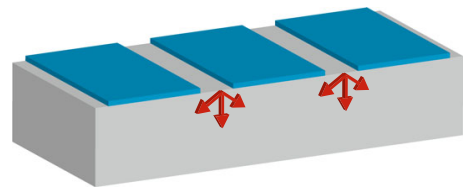

d
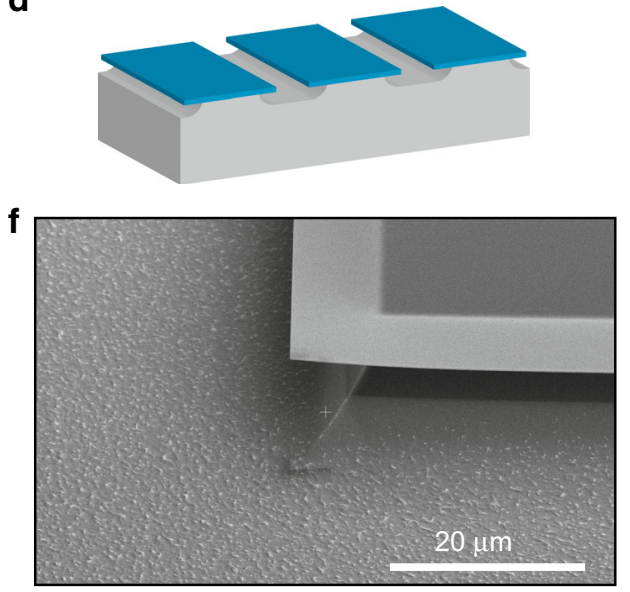

Fig. 4 Procedure for minimizing trench depth and width between the contact pads (blue). A layer of photoresist (purple) covers the full chip (a), and we etch only the sides of the chip, creating a tip overhang (b). The resist is then removed (c), and an additional very brief isotropic Si etch is used to create the trenches between the pads for electrical separation (d). SEM images of the finished device showing the undercut along a trench (e) and at a corner (f).

\section{FIB milling}

The minimum tip-to-tip distance previously achieved by having two separate SiN tips is $45 \mathrm{~nm}$ for a test device using $50 \mathrm{~nm} \mathrm{SiN} \mathrm{and} \mathrm{(sputtered)} 20 \mathrm{~nm} \mathrm{Au}^{16}$. Here, we work with a thicker and more reliable SiN film and $\mathrm{Au}$ layer. This also enlarges the radii of curvatures of the two tips and thereby limits the tip-to-tip distance to more than twice the radius, which is a similar limitation to bringing two individual tips into close proximity. The use of a $50 \mathrm{~nm}$ thick SiN and a very thin film reduces tip separation but causes uncertainties. First, depending on the length of the overhanging tips, the thin SiN tends to bend upwards due to the intrinsic stress of the film. While this bend is not a problem in principle, it might not always be equal for both tips when not connected. We therefore decided to use thicker $200 \mathrm{~nm} \mathrm{SiN}$ in combination with a slightly thicker evaporated Au film.

As a result of the excellent control we have over the shape of the tips, we create devices where the two apices are joined together at both the $\mathrm{SiN}$ and $\mathrm{Au}$ film and separate the tips using FIB milling. The aim is to mill only the metal layer and keep the SiN attached. A priori, this method presents several advantages: (i) FIB milling has the ability to achieve a higher resolution than $\mathrm{EBL}^{34}$, especially when combined with a $200 \mathrm{~nm}$ deep etch through the $\mathrm{SiN}$; (ii) the tip separation is not determined by the film thickness; (iii) the mechanical connection formed by the SiN eliminates possible height or tip-to-tip distance fluctuations, compared to those for earlier reported proof-of-principle devices ${ }^{16}$; and (iv) this approach improves the overall (macroscopic) stability of the tips. The dielectric breakdown voltage that can result in a short circuit between the two tips should occur well above $5 \mathrm{~V}$ for these structures and should not hinder normal STM operation. Another possibility for the separation of the metal we did not explore involves the use of electromigration to create a tip separation to possibly smaller than $5 \mathrm{~nm}^{35}$, although sufficient control over where the separation occurs might be challenging.

A well-known side effect of FIB milling is the addition of $\mathrm{Ga}^{+}$ions a few nanometers into the exposed top layer of the material. The impact of the ions can result in lattice defects, incorporated $\mathrm{Ga}$, and heat ${ }^{34}$. The range of added $\mathrm{Ga}^{+}$ions can vary from 10 to $100 \mathrm{~nm}$ in depth and 5 to $50 \mathrm{~nm}$ in width. The $\mathrm{Ga}^{+}$ions at the Au tip surface may be a cause for concern if they have an effect on the quality of the STM tips or their electronic properties; however, previous STM experiments have used tips modified by FIB before ${ }^{13,36}$. The difference here is that redeposition of the insulating $\mathrm{SiN}$ can occur. However, a short immersion in an HF solution or exposure to vapor $\mathrm{HF}^{37}$ can be used to remove a few nanometers of $\mathrm{SiN}$ without damaging the 
a
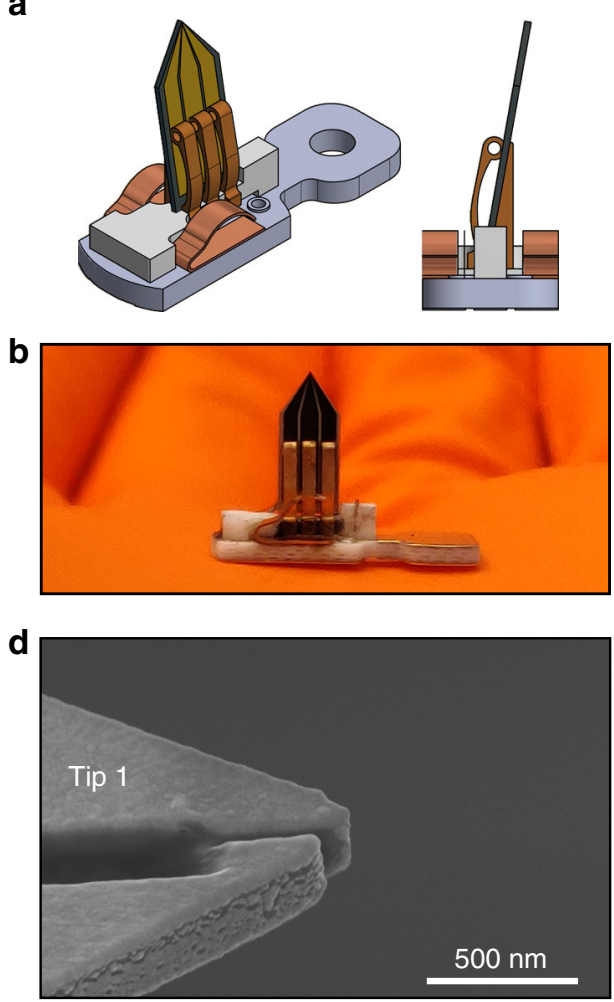
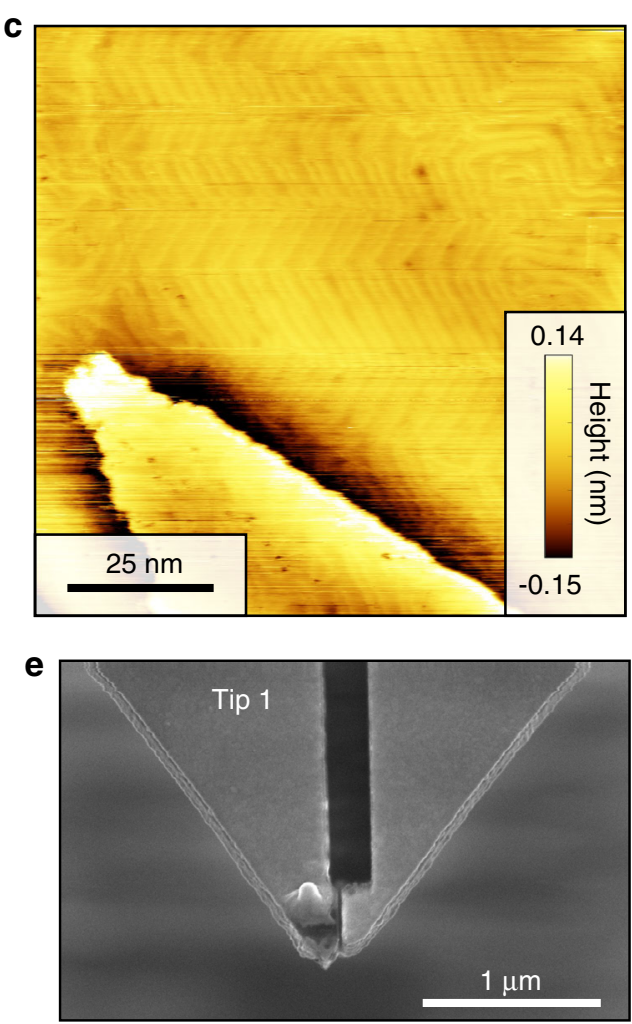

Fig. 5 Testing a fabricated device on a Au (111) surface. a Schematic drawing of a device holder including the double-tip device. $\mathbf{b}$ Photograph. c Topographic image of gold on a mica sample using our tip for STM imaging taken with setup conditions where $V_{b}=0.75 \mathrm{~V}$ and $I=50 \mathrm{pA}$. $\mathbf{d}$, e SEM micrograph of the device used to measure $(\mathbf{c})$ before and after applying a voltage of $>5 \mathrm{~V}$ between the two tips.

overall structure of the tips. Furthermore, a common in situ tip preparation technique called mechanical annealing $^{38,39}$ allows us to pick up clean gold from a Au (111) surface and may assist in attaining a clean STM tip and should assure tip sharpness. Variation of the tip-to-tip distance due to the graininess of the metal film may still occur but can be readily improved by optimization of the evaporation temperature; the use of thinner, less grainy films, such as AuPd; and additional shaping of the tip using FIB.

\section{Tip characterization}

To validate the fabrication method described in the paper, we test the device in a modified scanning tunneling microscope (STM) manufactured by RHK.

We built a custom holder for the device (Fig. 5a, b) consisting of three phosphor bronze contact clamps attached to an $\mathrm{Al}_{2} \mathrm{O}_{3}$ block on a base plate made out of a special printed circuit board equipped with vias to contact the STM. The device is then inserted into the holder and held by the contact springs. We insert the holders into our cryogenic ultrahigh vacuum STM.

For the imaging and breakdown experiments, we prepare a gold film with three cycles of $\mathrm{Ar}^{+}$sputtering. $\left(0.75 \mathrm{kV}, 3 \mathrm{~mA}\right.$ at $5 \times 10^{-5} \mathrm{mbar}$ for $\left.15 \mathrm{~min}\right)$ and subsequent annealing at $\sim 390^{\circ} \mathrm{C}$ for $30 \mathrm{~min}$ under UHV conditions. We then scan the gold on the mica sample. Figure $5 c$ shows a topographic image from the $\mathrm{Au}(111)$ surface exhibiting both step edges and typical herringbone reconstruction. A gold surface is commonly used to assess tip quality and stability; however, since it is metallic, gating effects from the second probe are not expected, as the potential drop occurs completely between the tips and sample. Finally, we investigate the breakdown voltage between the tips by applying voltages of $1 \mathrm{~V}, 2.5 \mathrm{~V}, 5 \mathrm{~V}$, and $7.5 \mathrm{~V}$ between the tips. The voltage is increased with the tip retracted, and the device is then tested on the $\mathrm{Au}$ (111) surface with the voltage fixed. The device continues to function up to $5 \mathrm{~V}$ applied between tips, but breaks down at $7.5 \mathrm{~V}$, as confirmed by SEM images (see Fig. 5d, e).

\section{Methods \\ Dicing}

The full dicing procedure is as follows. First, we dress the blade, load the chip and proceed to make a first cut and perform the hairline adjustment. Then, with a sequence of individually aligned dices as indicated in 
Fig. 3a, c, we cut out the chip shape. Line 1 is the one close to the tips and is therefore performed first. Line 2 follows and maintains the same alignment to ensure that when the chip stands upright, the bottom is exactly parallel to the top where the tips protrude. Cross lines 3 and 4 run through the shields typically around $20 \mu \mathrm{m}$ away from the tip on each side (Fig. 3b). Finally, we dice lines 5 and 6 parallel to each other and set the width of the chip to $3 \mathrm{~mm}$.

\section{FIB milling}

For the milling procedure, we load the chips on a piece of carbon tape into the FIB system with the sample holder firmly screwed in to prevent small displacements during tilting. We adjust the focus of the electron beam repeatedly as we move the stage to its working distance. We also correct for stigmatization and ensure that the electron beam is not shifted with respect to the ion beam for the alignment procedure. Then, we pick a reference point at high magnification and tilt the sample $52^{\circ}$ towards the ion beam while imaging with the electron beam. As soon as we image with the ion beam, the milling starts; therefore, we first find an area close to the tip but keep it outside the field of view. We can set up such that the fields of view of the electron and ion beam are the same and the magnifications are linked. Before we mill, we focus the ion beam at $\times 100,000$ magnification. Using the electron beam, we navigate to the tip, take a single scan with the ion beam and align the milling path. After the alignment, we typically take a second scan to check and compensate for a slight drift in the image. Then, we perform the milling process over a length from 1 to $2 \mu \mathrm{m}$ in less than a second using an optimized recipe for Au with a milling depth from 200 to $300 \mathrm{~nm}$.

\section{Conclusion}

We demonstrated a new fabrication procedure for integrated double-tip devices with a tip separation of $\sim 35 \mathrm{~nm}$ based on our highly flexible SiN-based smart tip platform. By etching the contact pads and two tip geometry into the SiN layer, we can create double-tip devices with an easy-to-implement process. The excellent mechanical and insulating properties of the SiN allow us to keep the tips attached via the $\mathrm{SiN}$ and utilize highresolution FIB milling of the metal layer to separate the two tips. This leads to increased mechanical stability, better alignment of the tips with respect to each other and removal of the need for any electromechanical actuation ${ }^{40}$.

The integration of these devices in existing commercial STM systems is straightforward when multiple tip contacts are available. The chips are only $200 \mu \mathrm{m}$ thick, can be made in various sizes and include large contact pads, making them well suited for the limited space available inside an STM head. Another benefit for the routine use of these tips is the ability to upscale the production of the devices to full wafer processing. By replacing the dicing of individual chips with a through-wafer Bosch etch, one could make a large number of chips from a single 4" wafer. For this, we would use a $\mathrm{SiO}_{2}$ hard mask combined with an electron beam or optical lithography to align the chip pattern to the underlying $\mathrm{SiN}$ tips/contacts with possibly even better accuracy compared to that of dicing. Once optimized, this is expected to yield faster, larger and more consistent production than current approaches.

A fully functioning double-tip STM based on the tips presented here differs significantly from a multiprobe system using metal wire tips. Here, the tips are joined at a fixed distance from each other on a chip that moves via a single piezoelectric tube positioning system. Consequently, the control over the two tips has unique challenges. To instigate tunneling in both tips, tilt stages to compensate for the slight difference in the length between the tips and a sample tilt are required. Based on the measurement of 4 different double-tips, we conclude that the typical angles between tips in various devices range from 4 to 15 degrees. After pretilting the sample or the devices, the final alignment for a double-tip experiment can be performed with a single piezoelectric element. As the 3D alignment is known from SEM images (see Fig. 1), one tilting stage is sufficient, while two stages would further increase the flexibility. Naturally, this results in a more advanced feedback scheme that takes the added tilt stage and both currents into account. Without a compact tilt stage, devices can be used in single-tip operation where the second tip, which is not tunneling, can be used as a gate to locally change the carrier density of dilute electron systems, such as semiconductor nanostructures ${ }^{28}$ or underdoped Mott insulators.

\section{Acknowledgements \\ We thank R.A. Norte for valuable discussions and acknowledge the support from the Kavli Nanolab Delft. This project was supported by the European Research Council (ERC StG Strong-Q and SpinMelt) and by the Netherlands Organization for Scientific Research (NWO/OCW), as part of the Frontiers of Nanoscience program, as well as through Vidi grants (680-47-536, 680-47-541).}

\section{Author contributions}

M.P.A. and S.G. conceived the idea and supervised the project. M.L. fabricated the samples; M.L., F.G., K.O., and T.B. built the STM; M.L., F.G., and K.O. made the tip holder and M.L. and T.B. performed the measurements. M.L., M.P.A., and S.G. wrote the manuscript.

Conflict of interest

The authors declare that they have no conflict of interest.

Received: 7 February 2020 Revised: 28 August 2020 Accepted: 1 September 2020

Published online: 02 November 2020 


\section{References}

1. Thamankar, R., Yap, T., Goh, K., Troadec, C. \& Joachim, C. Low temperature nanoscale electronic transport on the $\mathrm{MoS}_{2}$ surface. Appl. Phys. Lett. 103 083106 (2013).

2. Jaschinsky, P., Wensorra, J., Lepsa, M. I., Myslivečcek, J. \& Voigtländer, B. Nanoscale charge transport measurements using a double-tip scanning tunneling microscope. J. Appl. Phys. 104, 094307 (2008).

3. Hasegawa, S., Yoshimoto, S., \& Hobara, R. Device and Process Technologies for Microelectronics, MEMS, Photonics, and Nanotechnology N, Vol. 6800 (International Society for Optics and Photonics, 2008) p. 68000G.

4. Niu, Q., Chang, M. C. \& Shih, C. K. Double-tip scanning tunneling microscope for surface analysis. Phys. Rev. B 51, 5502-5505 (1995).

5. Byers, J. M. \& Flatté, M. E. Probing spatial correlations with nanoscale twocontact tunneling. Phys. Rev. Lett. 74, 306-309 (1995).

6. Settnes, M., Power, S. R., Petersen, D. H. \& Jauho, A.P. Theoretical analysis of a dual-probe scanning tunneling microscope setup on graphene. Phys. Rev. Lett. 112, 096801 (2014).

7. Settnes, M., Power, S. R., Petersen, D. H. \& Jauho, A.-P. Dual-probe spectroscopic fingerprints of defects in graphene. Phys. Rev. B 90, 035440 (2014).

8. Leeuwenhoek, M., Gröblacher, S., Allan, M. P. \& Blanter Y. M. Modeling Green's function measurements with two-tip scanning tunneling microscopy. Phys. Rev. B 102, 115416 (2020).

9. Khotkevych, N., Kolesnichenko, Y. \& van Ruitenbeek, J. Quantum interference effects in a system of two tunnel point-contacts in the presence of a single scatterer: simulation of a double-tip STM experiment. Low. Temp. Phys. 37, 53-58 (2011)

10. Gramespacher, T. \& Büttiker, M. Quantum shot noise at local tunneling contacts on mesoscopic multiprobe conductors. Phys. Rev. Lett. 81, 2763-2766 (1998).

11. Gramespacher, T. \& Büttiker, M. Local densities, distribution functions, and wave-function correlations for spatially resolved shot noise at nanocontacts. Phys. Rev. B 60, 2375-2390 (1999).

12. Hasegawa, S. Multi-probe scanning tunneling microscopy, in Scanning Probe Microscopy: Electrical and Electromechanical Phenomena at the Nanoscale, (edited Kalinin S \& Gruverman A) 480-505 (Springer New York, New York, NY, 2007).

13. Kolmer, M. et al. Two-probe STM experiments at the atomic level. J. Phys. Condens. Matter. 29, 444004 (2017).

14. Okamoto, H. \& Chen, D. An ultrahigh vacuum dual-tip scanning tunneling microscope operating at 4.2 K. Rev. Sci. Instrum. 72, 4398-4403 (2001).

15. Kolmer, M. et al. Electronic transport in planar atomic-scale structures measured by two-probe scanning tunneling spectroscopy. Nat. Commun. 10, 1573 (2019).

16. Leeuwenhoek, M. et al. Nanofabricated tips for device-based scanning tunneling microscopy. Nanotechnology 30, 335702 (2019).

17. Jaschinsky, P., Coenen, P., Pirug, G. \& Voigtländer, B. Design and performance of a beetle-type double-tip scanning tunneling microscope. Rev. Sci. Instrum. 77, 093701 (2006)

18. Yang, J., Sordes, D., Kolmer, M., Martrou, D. \& Joachim, C. Eur. Phys. J. Appl. Phys. 73, 10702 (2016).

19. V. Cherepanov, et al. In Atomic Scale Interconnection Machines 9-21. (Springer, 2012).
20. Roychowdhury, A. et al. A 30 mK, 13.5 T scanning tunneling microscope with two independent tips. Rev. Sci. Instrum. 85, 043706 (2014).

21. Ge, J.-F. et al. Development of micro-four-point probe in a scanning tunneling microscope for in situ electrical transport measurement. Rev. Sci. Instrum. $\mathbf{8 6}$ 053903 (2015).

22. Pan, S., Hudson, E. W. \& Davis, J. ${ }^{3}$ He refrigerator based very low temperature scanning tunneling microscope. Rev. Sci. Instrum. 70, 1459-1463 (1999).

23. Vettiger, P. et al. The "Millipede"-More than thousand tips for future AFM storage. IBM J. Res. Dev. 44, 323-340 (2000).

24. de Graaf, S. E., Danilov, A., Adamyan, A. \& Kubatkin, S. A near-field scanning microwave microscope based on a superconducting resonator for low power measurements. Rev. Sci. Instrum. 84, 023706 (2013).

25. Ge, J.-Y. et al. Nanoscale assembly of superconducting vortices with scanning tunnelling microscope tip. Nat. Commun. 7, 13880 (2016).

26. Mouaziz, S., Boero, G., Popovic, R. S. \& Brugger, J. Polymer-Based Cantilevers With Integrated Electrodes. J. Microelectromech. Syst. 15, 890-895 (2006).

27. Huber, M. E. et al. Gradiometric micro-SQUID susceptometer for scanning measurements of mesoscopic samples. Rev. Sci. Instrum. 79, 053704 (2008).

28. Gurevich, L., Canali, L. \& Kouwenhoven, L. P. Scanning gate spectroscopy on nanoclusters. Appl. Phys. Lett. 76, 384-386 (2000).

29. Siahaan, T., Kurnosikov, O., Barcones, B., Swagten, H. \& Koopmans, B. Cleaved thin-film probes for scanning tunneling microscopy. Nanotechnology 27, 03LT01 (2015).

30. Xu, Y., MacDonald, N. \& Miller, S. Integrated micro-scanning tunneling microscope. Appl. Phys. Lett. 67, 2305-2307 (1995).

31. Nagase, M., Hibino, H., Kageshima, H. \& Yamaguchi, H. In-plane conductance measurement of graphene nanoislands using an integrated nanogap probe. Nanotechnology 19, 495701 (2008).

32. Ciftci, H. T., Van, L. P., Koopmans, B. \& Kurnosikov, O. Polymer patterning with self-heating atomic force microscope probes. J. Phys. Chem. A 123, 8036-8042 (2019).

33. Norte, R. A., Forsch, M., Wallucks, A., Marinković, I. \& Gröblacher, S. Platform for measurements of the Casimir force between two superconductors. Phys. Rev. Lett. 121, 030405 (2018).

34. Volkert, C. A. \& Minor, A. M. Focused ion beam microscopy and micromachining. MRS Bull. 32, 389-399 (2007).

35. Heersche, H. B., Lientschnig, G., O'Neill, K., van der Zant, H. S. \& Zandbergen, H. W. In situ imaging of electromigration-induced nanogap formation by transmission electron microscopy. Appl. Phys. Lett. 91, 072107 (2007).

36. Schmucker, S. et al. Field-directed sputter sharpening for tailored probe materials and atomic-scale lithography. Nat. Commun. 3, 935 (2012).

37. Williams, K. R., Gupta, K. \& Wasilik, M. Etch rates for micromachining processing-part II. J. Micro- electromech. Syst. 12, 761-778 (2003).

38. Castellanos-Gomez, A. et al. Highly reproducible low temperature scanning tunneling microscopy and spectroscopy with in situ prepared tips. Ultramicroscopy 122, 1-5 (2012).

39. Tewari, S. et al. Robust procedure for creating and characterizing the atomic structure of scanning tunneling microscope tips. Beilstein J. Nanotechnol $\mathbf{8}$, 2389 (2017).

40. Martin, C. A., Smit, R. H., van der Zant, H. S. \& van Ruitenbeek, J. M. A nanoelectromechanical single-atom switch. Nano Lett. 9, 2940-2945 (2009). 